\title{
Adaptação Transcultural: Multidimensional Orientation Toward Dying and Death Inventory (MODDI-F) à realidade brasileira Cross Cultural Adaptation: Multidimensional Orientation Toward Dying and Death Inventory (MODDI-F) to the Brazilian Reality
}

\author{
Marcos Antônio de Olivas \\ Mestre em Bioética. Especialista em Docência do Ensino Superior \\ Docente da FEPI do Centro Universitário de Itajubá e Docente da \\ Escola de Enfermagem Wenceslau Braz. \\ Endereço: Rua Prof. Estácio Tavares de Melo, 229, Varginha, CEP \\ 37501-138, Itajubá, MG, Brasil. \\ E-mail: olivasळitacabo.com.br \\ José Vitor da Silva \\ Pós-Doutor em PBioética. Diretor Acadêmico da Escola de Enfer- \\ magem Wenceslau Braz. Docente na Faculdade de Enfermagem \\ de Itajubá e na Univesidade do Vale do Sapucaí. \\ Endereço: Rua João Faria Sobrinho, 61 - ap 301, Varginha, CEP \\ 37501-080, Itajubá, MG, Brasil. \\ E-mail: enfjvitorsilvaœoi.com.br \\ Franklin Santana Santos \\ Doutor em Medicina. Pós-Doutor em Psicogeriatria. Coordenador \\ dos Cursos de Pós-graduação lato sensu de Tanatalogia e Cuidados \\ Paliativos da Universidade Santa Cecília/Pinus Lonaeva. \\ Endereço: Rua Apinajés, no 268 apt 112 Perdizes, CEP 05017-000, \\ São Paulo, SP, Brasil. \\ E-mail: franklinssantosळhotmail.com
}

\section{Resumo}

Muitos são os estudos realizados sobre a morte, entretanto, não existem na realidade brasileira instrumentos disponíveis sobre esse fenômeno. Este estudo teve como objetivo realizar a adaptação transcultural do Multidimensional Orientation Toward Dying and Death Inventory (MODDI-F) - Inventário de Orientação Multidimensional em Relação ao Morrer e à Morte (IMMOR); originalmente elaborado na Alemanha e traduzido para o inglês, está constituído por 47 itens divididos em oito domínios. Essa escala avalia as reações e atitudes das pessoas em relação ao medo da morte e do morrer. Para se adquirir a primeira versão, foi realizada a sua tradução para a língua portuguesa, separadamente, por três peritos em inglês. A primeira versão foi submetida ao corpo de cinco juízes para a "Avaliação das Equivalências Semântica e Idiomática" da qual se obteve a segunda versão, que foi submetida ao segundo grupo de cinco juízes para avaliação conceitual e cultural ( $3^{\text {a }}$ versão). A seguir desenvolveu-se o Grupo Focal, no qual o IMMOR foi analisado por representantes da comunidade, de ambos os gêneros, diferentes faixas etárias e graus de escolaridade, estabelecendo-se a quarta versão, que foi submetida à back-translation e enviada ao autor. Após os ajustes sugeridos por ele, alcançou-se a versão final. De acordo com as etapas metodológicas desenvolvidas, considera-se o presente instrumento adaptado à cultura brasileira.

Palavras-chave: Tradução; Adaptação Transcultural; Morte; Morrer. 


\section{Abstract}

Many studies have been conducted about death; however, there are no instruments available in Brazil to evaluate this phenomenon. The purpose of this study was to perform the cross-cultural adaptation of the Multidimensional Orientation Toward Dying and Death Inventory (MODDI-F), originally designed in Germany and translated into English, constituted by 47 items divided in eight domains. This scale evaluates people's reactions and attitudes in relation to fear of death and dying. To produce the first version, it was translated into Portuguese, separately, by three experts in English. The first version was submitted to five judges for the "Semantic and Idiomatic Equivalences Evaluation", which produced the second version. The second version was submitted to the second group of five judges for a conceptual and cultural evaluation (third version). Then the Focal Group was developed, in which the instrument, which was called IMMOR in Portuguese, was analyzed by community representatives, of both sexes, different ages and levels of schooling, establishing the fourth version, which was submitted to a back-translation and sent to the author. After the implementation of the changes he suggested, we reached the final version. According to the methodological steps that were developed, it is possible to state that the present instrument is adapted to the Brazilian culture.

Keywords: Translation; Cross-cultural Adaptation; Dying; Death.

\section{Introdução}

Ao longo da história, a vida, a morte e o sofrimento sempre promoveram debates na área da Filosofia, estendendo-se para a ética, bioética e moral. Aquela ciência e seus ramos buscam desvendar, de alguma forma, o significado da vida e da morte, inserido numa determinada cultura que, mediante os valores vivenciados, acabam por interferir no processo de morrer (Fernandes e Freitas, 2006).

Mesmo sendo senso comum dizer "que a morte faz parte da vida e é a única certeza que se tem”, a forma de encará-la foi modificada com o decorrer do tempo e, atualmente, este fenômeno causa certo pavor (Simão, 2010).

Em nossa cultura, nega-se a morte e muitos querem permanecer jovens. As pessoas não se preparam para aceitar a morte como um processo natural da vida. Desse modo, torna-se também muito difícil para uma pessoa acompanhar um paciente que a vivencia, uma vez que ela não aceita a morte de outro, nem a sua (Fernandes e Freitas, 2006).

Pensar em morte, na cultura ocidental, é pensar em dor, pois a morte está associada a um sofrimento intenso e doloroso. Tem-se como um dos elementos essenciais do sofrimento a dor. Assim, é comum os médicos, ao se referirem à morte súbita, expressarem como "Morte sem sofrimento", ou seja, aguda que não determina sofrimento ao paciente, principalmente relacionado à dor e à angústia. (Silva e Simões, 2010).

Segundo Feitosa (2011, p. 29):

Sabemos ser antiga a interferência humana no momento da morte, mas tal ingerência ganhou especial relevância em face da intensa evolução biotecnológica ocorrida na segunda metade do século XX. Em nossos dias, é possível prolongar artificialmente a existência de um doente, ainda que a Medicina não lhe possa oferecer nenhuma expectativa de cura ou maior conforto em tal prolongamento.

Mais de $75 \%$ das pessoas morrem nos hospitais, porque não há mais condições para cuidar de doentes ou pessoas idosas nos seus domicílios. As diversas dificuldades familiares obrigam as pessoas a se afastarem cada vez mais de seus lares. Então, o 
hospital torna-se a alternativa eleita (Barchifontaine e Pessini, 2002).

Por outro lado, a pessoa que está diante da morte e hospitalizada tem medo do abandono da família, medo dos procedimentos médicos e medo de morrer. A família é muito importante para o paciente, trazendo-lhe a sensação de segurança diante de tantos acontecimentos desconhecidos. Entretanto, esta mesma família também é acometida de medos, angústias e não sabe como proceder diante do seu familiar no leito de morte (Esslinger, 2004).

A morte está em todas as partes, mas não se fala dela. Está presente na vida tensa das cidades anônimas, mas continua sendo banalizada e inapropriado falar da morte e do morrer, assim como era inadequado falar de determinados prazeres, estigmas ou de sexo (Drumond e Stepke, 2007).

Porém, para lutar contra a morte é preciso integrá-la na mais íntima dimensão da vida. Negar a morte significa adotá-la como castigo e não como lei natural e necessária da vida que consiste em renovação (Bellato e Carvalho, 2005).

Assim, não há mais espaço no mundo contemporâneo para tantas inquietações diante dela. Ela precisa ser amplamente discutida, estudada e interpretada para que possa ser melhor entendida e aceita.

Ao se consultar as diversas bases de dados, observou-se um número considerável de pesquisas sobre temas relacionados à morte, como: o processo de morte e morrer, paciente fora de possibilidade terapêutica, humanização do final da vida e cuidados paliativos. (Kübler-Ross, 1977; Leloup, 2001; Barchifontaine e Pessini, 2002; Kovács, 2003; Esslinger, 2004; Mccouchlan, 2004; Py e Oliveira, 2004; Bellato e Carvalho, 2005; Fernandes e Freitas, 2006; Bretas e col., 2006; Silva e Simões, 2008; Doyle, 2009).

Entretanto, constatou-se que trabalhos sobre medo da morte e do morrer são ainda muito escassos. Sobre esse tema, encontrou-se apenas uma pesquisa, que é a de Wittkowski (2001), o autor da escala: Multidimensional Orientation Toward Dying And Death Inventory (MODDI-F).

O contato com o assunto despertou neste autor o interesse pelo tema, sobretudo ao constatar que, na realidade brasileira, não existiam instrumentos de medida sobre morte e especificamente sobre o medo da morte e do morrer.

Segundo Kimura (1999), na atualidade, questiona-se sobre a necessidade de se criar novos instrumentos de medida, quando já se dispõe de outros com o mesmo objetivo e qualidade comprovada já que o processo de elaboração é muito mais dispendioso não só em tempo como também em empenho pessoal e financeiro.

Guillemin e colaboradores (1993) ressaltam que, ao adaptar e validar uma medida pré-existente para o contexto cultural de uma população-alvo, têm-se as seguintes vantagens: fornece uma medida padrão para uso em estudos internacionais; permite a inclusão de imigrantes, evitando o preconceito frequente de representar apenas a cultura dominante do país; permite a comparação entre grupos culturais nacionais contando com uma medida padrão projetada e adaptada para medir o fenômeno transcultural; proporciona menor custo e consumo de tempo do que criar uma nova medida. Contudo, deve-se ter em mente que a adaptação transcultural também requer uma atenção cuidadosa e o envolvimento de muitas pessoas.

No entanto, faz-se necessário um processo de tradução e adaptação abrangentes para que tais instrumentos sejam utilizados em diferentes realidades socioculturais, na tentativa de alcançar uma equivalência cultural, com posterior estudo de sua validade nessa nova população (Vilete e col., 20o6).

Assim, a adaptação transcultural do instrumento Multidimensional Orientation Toward Dying And Death Inventory (MODDI-F), já validado originariamente, mostra-se útil para avaliar um quadro bastante abrangente de atitudes ou reações diante da morte, como também oferece subsídios aos pesquisadores para delineamento de outras pesquisas sobre esse assunto.

Paralelamente a tudo isso, a importância deste estudo foi fundamental também no sentido de estabelecer, reforçar ou ampliar os conhecimentos a respeito da morte e do morrer, percebendo-os como eventos naturais e sobre os quais se precisa discutir e aprender a lidar. 


\section{Método}

\section{Delineamento do Estudo e Instrumento}

0 presente estudo foi do tipo metodológico e descritivo.

Considerando que o MODDI-F se refere a uma escala com abordagem completa ao tema Morte e Morrer, assim como a sua grande utilização nos Estados Unidos, optou-se por sua escolha, que será útil no sentido de avaliar os diversos aspectos relacionados com a morte e o morrer.

Originalmente, o MODDI-F foi desenvolvido no idioma alemão (Wittkowski, 2001). Entretanto, este autor o liberou em inglês, sem motivos aparentes, para ser utilizada nos Estados Unidos. Da mesma forma, quando se solicitou autorização para uso do MODDI-F, ele o enviou aos autores deste estudo, em inglês.

Quanto à fundamentação conceitual do MODDI-F, ele está baseado na premissa de que a pessoa ao avaliar o medo da morte o faz também em relação ao medo de morrer. Além do mais, o medo de um lado e a aceitação do outro, são consideradas as maneiras gerais mais importantes de pensar e sentir com respeito ao morrer e à morte (Wittkowski, 2001).

O MODDI-F é de natureza multidimensional, formado por 47 itens, divididos em oito domínios (Quadro 1). A pontuação mínima é de 47 pontos e o escore máximo, correspondente a 188 pontos, sendo que os itens 9 e 38 apresentam pontuação invertida.

\section{Quadro I - Subescalas da MODDI-F e seus itens correspondentes}

\begin{tabular}{|l|l|}
\hline 1 - Medo do momento da própria morte (FODy-F) & $1,8,16,23,31,37,43,46$ \\
\hline 2 - Medo da própria morte (FODe-F) & $5,13,20,28,35,42$ \\
\hline 3- Medo do momento da morte de outra pessoa (FOPDy-F) & $3,10,18,25,33,39$ \\
\hline 4 - Medo da morte de outra pessoa (FOPDe-F) & $7,15,22,30$ \\
\hline 5 - Medo de cadáveres (FC-F) & $12,27,41,45$ \\
\hline 6 - Aceitação do momento da própria morte e da própria própria morte (AODD-F) & $4,11,19,26,34,40,44,47$ \\
\hline 7 - Aceitação da morte de outra pessoa (AOPDe-F) & $2,9,17,24,32,38-$ \\
\hline 8- Rejeição da própria morte (RODe-F) & $6,14,21,29,36$ \\
\hline
\end{tabular}

*** Os itens 9 e 38 são pontuados em sentido contrário.

\section{Aspectos Éticos da Pesquisa}

O presente estudo foi submetido e aprovado pelo Comitê de Ética em Pesquisa da Universidade Vale do Sapucaí, da cidade de Pouso Alegre, M.G. conforme Parecer Consubstanciado, sob Protocolo $\mathrm{n}^{\circ}$ 1223/10.

\section{Desenvolvimento do estudo}

O desenvolvimento da adaptação transcultural do MODDI-F seguiu as seguintes etapas metodológicas: tradução do instrumento de sua língua original para a língua portuguesa brasileira; avaliação das equivalências semântica e idiomática entre a versão original e a versão traduzida; avaliação das equivalências conceitual e cultural da segunda versão originada das equivalências semântica e idiomática; realização do grupo focal e da back-translation. (Figura 1)

O período de realização do estudo compreendeu de abril de 2009 a outubro de 2010. Especificamente, a fase de equivalência semântica idiomática e conceitual, assim como a sessão de grupo focal. 
Figura I - Diagrama do desenvolvimento da adaptação cultural do MODDI-F (Adaptado de GUILLEMIN, 1995 e de GUTIERREZ, 2000)
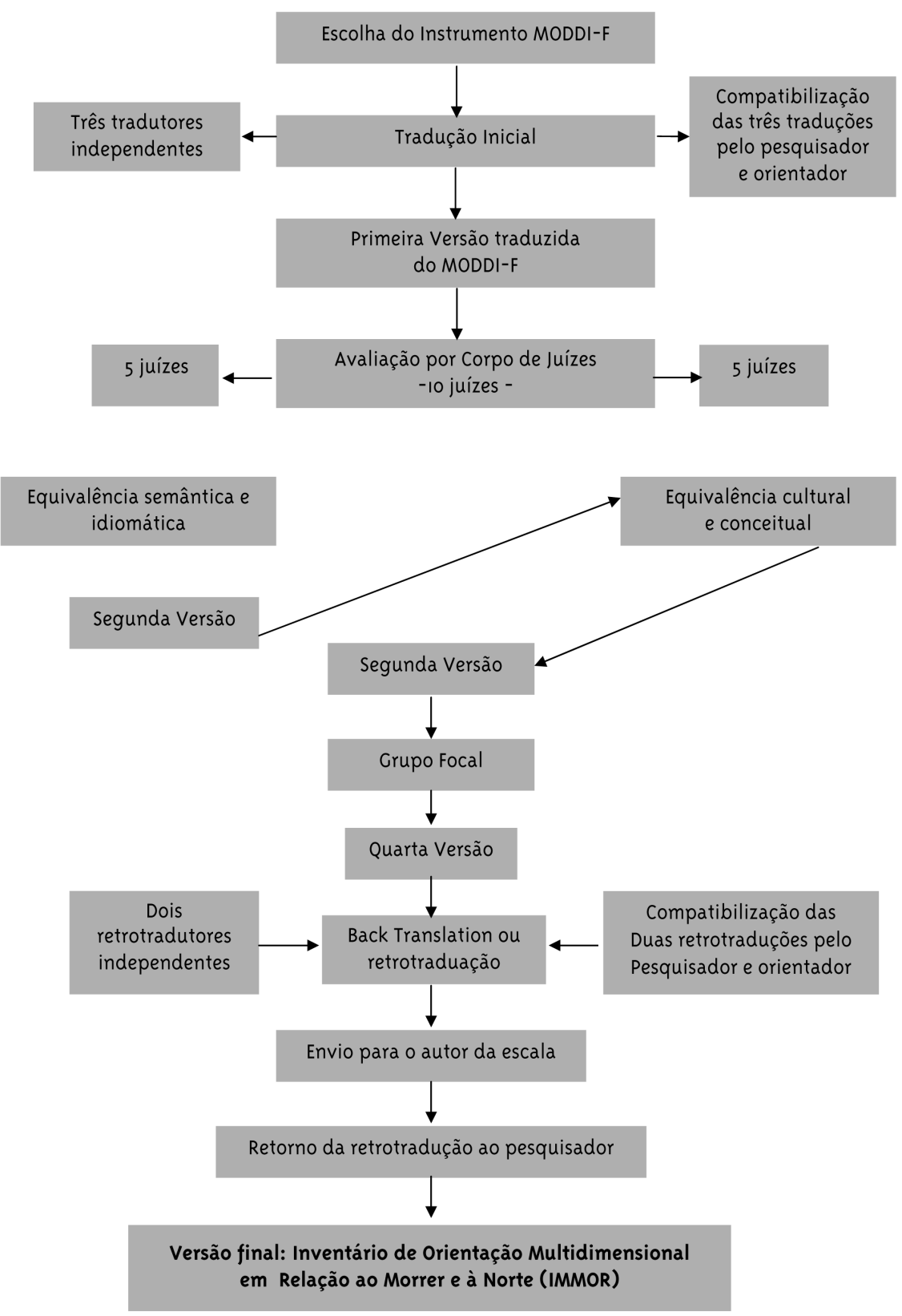


\section{Resultados e Discussão}

Os resultados do presente estudo consistem na apresentação das etapas que estabeleceram a adaptação transcultural do Multidimensional Orientation Toward Dying and Death Inventory - MODDI-F à cultura brasileira, que são as seguintes:

\section{A tradução do Multidimensional Orientation To- ward Dying and Death Inventory (MODDI-F)}

A tradução do MODDI-F para a língua portuguesa foi realizada, separadamente, por três peritos em inglês. Para a realização da tradução do instrumento foram selecionados três professores de inglês com larga experiência em docência da Língua Inglesa em escolas de línguas localizadas na cidade de Itajubá-MG. As traduções procuraram considerar os aspectos linguísticos e o significado atribuído aos termos em nossa realidade. Posteriormente, já em posse das traduções, o pesquisador e o orientador se reuniram e as três versões traduzidas foram analisadas item por item. Dentre as três traduções, procuraram escolher os itens com linguagem mais próxima à realidade brasileira. A partir da análise das três traduções, foi elaborada outra versão denominada "primeira versão traduzida do Multidimensional Orientation Toward Dying and Death Inventory - MODDI-F”.

\section{Avaliação por corpo de juízes}

A primeira versão elaborada pelo pesquisador e pelo orientador foi submetida a um corpo de cinco juízes, aos quais foi solicitada a avaliação das versões original e final do instrumento ( $1^{\underline{a}}$ versão traduzida do MODDI-F). O Corpo de Juízes foi constituído de cinco brasileiros, peritos em inglês com bacharelado em Letras e vasto conhecimento das línguas inglesa e portuguesa. Foi explicado, pelo pesquisador, aos avaliadores, o processo da avaliação e a importância de suas contribuições para o desenvolvimento da adaptação cultural do instrumento. Esse grupo de juízes efetuou a análise das equivalências semântica e idiomática dos itens do instrumento.

Após a devolução das avaliações do instrumento pelo corpo de juízes, foi realizada, pelo pesquisador e pelo orientador, a análise dos itens e realizadas as alterações sugeridas pelos avaliadores.

Dos 47 itens avaliados, os de números 1, 5, 9, 13, $16,17,18,19,21,22,29,37,39,41,42,4344,45$ e 46 foram tidos como equivalentes, não recebendo, dessa forma, quaisquer sugestões de alteração.

Os itens 2, 3, 6, 7, 8, 10, 12, 14, 15, 20, 23, 24, 25, 26, $27,28,30,31,32,33,34,35,36,38$ e 4 o foram avaliados como não equivalentes e receberam sugestões de alterações pelo corpo de juízes, que foram acatadas pelo pesquisador.

Os itens 4, 11, 16 e 47 receberam do próprio pesquisador as seguintes alterações: no item 4 , a expressão: "em relação ao processo de morrer" foi modificada para "em relação à morte". No item 11, a expressão: "o processo de morte" foi modificada para "a morte". No item 16, a expressão: "um processo lento de morte" foi modificada para "o processo lento do morrer". No item 47, a expressão: "processo de morte" foi modificada para "processo do morrer". Estas alterações, mesmo não sendo sugeridas pelo corpo de juízes, mostraram-se necessárias em decorrência de seus significados, pois MORRER é o processo e MORTE é o produto, conforme estabelece essa diferença Santos (2009).

A partir disso, foi constituída a segunda versão traduzida do MODDI-F.

A segunda versão foi encaminhada a outro comitê, constituído também por cinco juízes, para a análise das equivalências cultural e conceitual dos itens. Para compor esse comitê de especialistas, foram escolhidos: profissionais das áreas de saúde e humanas; com domínio da língua inglesa, conhecimento dos processos de construção e adaptação de instrumentos de medida de variáveis psicossociais e conhecimento sobre a temática "morte e morrer".

Foram considerados equivalentes do ponto de vista conceitual, os itens que apresentaram, no mínimo, 80\% de equivalência entre os avaliadores. Aqueles que apresentaram nível de equivalência inferior a este foram modificados pelo autor do presente estudo.

Dos 47 itens avaliados, os itens 1, 3, 5, 6, 7, 8, 9, $10,12,14,15,18,19,22,23,24,25,30,31,33,34,37$, 39, 40, 41, 42, 43, 45, 46 e 47 receberam, do corpo de juízes, nível de concordância mínima de 80\%, portanto não sofreram modificações.

Apresentaram nível de equivalência inferior a $80 \%$ os itens $2,4,11,13,16,17,20,26,27,28,29,32$, $35,36,38,44$, os quais foram modificados conforme sugestões apresentadas. 
Para obter o índice de concordância entre os juízes quanto à equivalência conceitual dos itens, foi adotado o modelo seguido por Kimura (1999).

IVC $=n^{\circ}$ de itens avaliados como equivalentes por 2 juízes Total de itens de escala

- onde IVC + Índice de Validade de conteúdo

$$
V C=\frac{46}{47}=0,9787
$$

As avaliações de cada juiz foram contrastadas com as avaliações de todos os demais, calculando-se o IVC para cada par (juiz A x juiz B; juiz A x juiz C; juiz $B \times$ juiz $C$; juiz $B \times$ juiz $D$; e assim sucessivamente).

O resultado das análises das equivalências cultural e conceitual permitiu a elaboração da terceira versão traduzida do MODDI-F.

Após esta etapa, definiu-se o Inventário de Orientação Multidimensional em Relação ao Morrer e à Morte. 0 pesquisador e o seu orientador juntamente decidiram pela adoção da sigla IMMOR para denominar o instrumento traduzido.

\section{Grupo focal}

O pesquisador e o orientador submeteram o IMMOR à avaliação de um grupo da comunidade de Itajubá, MG, que, metodologicamente, denomina-se Grupo Focal (GF), formado por cinco pessoas de ambos os gêneros, idade entre 20 e 80 anos e grau de escolaridade variado. $\mathrm{O}$ objetivo do GF foi avaliar a compreensão de cada item do instrumento em questão.

Para a reunião do GF, foram adotados os seguintes procedimentos:

No dia, horário e local agendados, os participantes foram recebidos pelo pesquisador e orientador, que explicaram o processo metodológico da adaptação transcultural e, especificamente, do instrumento a ser analisado.

Em seguida, com a utilização do data show, os itens do instrumento foram projetados, um a um, para que os participantes os analisassem.

Dos 47 itens analisados pelos participantes, por consenso do grupo, foram considerados como "de fácil compreensão", não sofrendo, dessa forma, quaisquer modificações.
Após a reunião do GF, embora os itens não sofressem quaisquer alterações, considerou-se sua realização como a obtenção da quarta versão do IMMOR.

\section{Back-translation ou retrotradução}

É importante enfatizar que, na maioria dos trabalhos sobre adaptação cultural, a back-translation é realizada após a tradução da escala (1 $1^{\underline{a}}$ Versão). Entretanto, no estudo realizado por Silva e Kimura (2002), a autora da escala que estava sendo adaptada à língua portuguesa solicitou que a back-translation fosse realizada após a obtenção da versão originada das equivalências conceitual e cultural. Associados a isto, encontraram-se também os estudos de Vilete e colaboradores (2006) e Laranjeira (2008), nos quais a back-translation foi efetuada a partir da realização dos grupos focais e por isso optou-se neste trabalho que a referida etapa fosse realizada após o GF.

De posse da quarta versão originada do GF, a escala foi submetida à "back-translation", retrotradução, do português ao inglês.

A finalidade da retrotradução foi de verificar se existiam divergências no significado e no conteúdo das versões original e traduzida da escala. Por esse motivo, os dois retrotradutores não foram orientados quanto aos objetivos e conceitos envolvidos no conteúdo do material. Diante disto a "back-translation" foi efetuada de forma independente.

Para a escolha dos back-translators foram considerados os seguintes critérios: os tradutores deviam ser nativos de países de língua inglesa; fluentes nas formas coloquiais da língua de origem e com domínio da língua portuguesa.

A partir das duas retrotraduções, a convite do pesquisador, um outro tradutor, norte-americano, professor de inglês e com domínio da língua portuguesa, realizou a versão final da back-translation. Para tanto, após a análise das retrotraduções, manteve-se os itens considerados mais adequados e se efetuou, nos demais, os ajustes necessários.

Após a obtenção da versão final da back-translation, a escala foi enviada ao Dr. Wittkowski, que, após tomar conhecimento da metodologia utilizada na tradução da escala do inglês para o português e da "back-translation", revisou-a e apresentou sugestões. 
Para efetuar os ajustes indicados pelo Dr. Joachim Wittkowski, o pesquisador e o orientador reuniram-se novamente com o norte-americano e, após considerações e análises, realizaram as modificações apontadas.

O autor da escala também apresentou a sugestão de manter as quatro opções originais de respostas (escala Likert) e não cinco, como havia sido sugerido pelo corpo de juízes. Tal sugestão foi acatada pelo pesquisador para manter a estrutura original de respostas do instrumento.

Após esses ajustes, obteve-se outra versão da back-translation, que foi novamente enviada ao autor da escala, adquirindo-se a sua concordância.

Mediante as modificações adotadas para a realização da segunda versão da back-translation, efetuaram-se também algumas modificações na quarta versão da escala adaptada culturalmente, da qual se originou a quinta e última versão do instrumento.

\section{Considerações Finais}

O Multidimensional Orientation Toward Dying and Death Inventory (MODDI-F) encontra-se devidamente adaptado à cultura brasileira, após o desenvolvimento das diversas etapas metodológicas consignadas por: tradução, equivalências semântica - idiomática e conceitual - cultural, grupo focal e back-translation.

A escolha do Inventário de Orientação Multidimensional em Relação ao Morrer e à Morte (IMMOR) baseou-se na necessidade de disponibilizar, à realidade brasileira, instrumento de medida devidamente adaptado a esta cultura, que possa avaliar os aspectos teóricos da morte e do morrer. Outro fator preponderante para tal escolha refere-se à inexistência na realidade brasileira de instrumentos de medida que possam mensurar as atitudes e reações das pessoas em relação à morte e ao morrer. A decisão da escolha desta escala deve-se ao fato de se tratar de um instrumento desenvolvido dentro de rigorosos critérios metodológicos e já validado em outras culturas.

A Escala de Orientação Multidimensional em Relação à Morte e ao Morrer (IMMOR) mostrou-se de fácil compreensão e poderá ser útil para avaliar um quadro bastante abrangente de atitudes ou reações diante da morte e do morrer, como também oferecer subsídios aos pesquisadores para delineamento de outras pesquisas sobre esse assunto.

\section{Referências}

BARCHIFONTAINE, C. de P. de; PESSINI, L. Bioética: alguns desafios. São Paulo: Loyola, 2002. BELLATO, R.; CARVALHO, E. C. O jogo existencial e a ritualização da morte. Revista LatinoAmericana de Enfermagem, Ribeirão Preto, v. 13, n. 1, 2005. Disponível em: <http://www.scielo. br/scielo.php?script=sci_arttext\&pid=So104$692005000100016 \& \operatorname{lng}=\& n r m=i s 0>$. Acesso em: 10 out. 2011.

BRETAS, J. R. S.; OLIVEIRA, J. R.; YAMAGUTI, L.. Reflexões de estudantes de enfermagem sobre morte e o morrer. Revista da Escola de Enfermagem da USP, São Paulo, v. 40, n. 4, 2006 . Disponível em: <http://www.scielo. br/scielo.php?script=sci_arttext\&pid=Soo8o$62342006000400005 \& \mathrm{lng}=\& \mathrm{nrm}=\mathrm{iso}>$. Acesso em: 10 Out. 2011.

DOYLE, D. Bilhete de plataforma: vivências em cuidados paliativos. Tradução de M. T. A. Figueiredo; M. G. M. C. A. Figueiredo. São Caetano do Sul, SP: Difusão Editora, 2009.

DRUMOND, J. G. F.; STEPKE, F. L. Fundamentos de uma antropologia bioética: o apropriado, o bom e o justo. São Paulo: Loyola, 2007.

ESSLINGER, I. O paciente, a equipe de saúde e o cuidador: de quem é a vida, afinal? Um estudo acerca do morrer com dignidade. In: PESSINI, L.; BERTACHINI, L. Humanização e cuidados paliativos. São Paulo: Loyola, 2004. p.149-66.

FEITOSA, E. N. A terminalidade da vida em pacientes pediátricos: desafios bioéticos e análise de um caso. In: SALLES, A. A. Bioética - velhas barreiras, novas fronteiras. Belo Horizonte: Mazza, 2011. p. 17-42.

FERNANDES, M. de F. P.; FREITAS, G. F. de. Processo de morrer. In: OGUISSO, T.; ZOBOLI, E. (Org.). Ética e bioética: desafio para a enfermagem e a saúde. São Paulo: Manole, 2006. p. 153-69. 
GUILLEMIN, F.; BOMBARDIER, C.; BEATON, D. Cross cultural adaptation of health related quality of life measures: literature review and proposed guidelines. Journal of Clinical Epidemioly, Toronto on Canadá, v. 46, n. 12, p. 1417-32, 1993.

KIMURA, M. Tradução para o português e validação do "Quality of life index of ferrans and powers”. 1999. Tese (Doutorado em Enfermagem) - Escola de Enfermagem da Universidade de São Paulo, São Paulo.

KOVÁCS, M. J. Educação para a morte - desafio na formação de profissionais de saúde e educação. São Paulo; Casa do Psicólogo: Fapesp, 2003.

KÜBLER-ROSS, E. Sobre a morte e o morrer. Tradução de T. L. Kipnis. São Paulo. Edart, 1977.

LARANJEIRA, C. E. Tradução e validação portuguesa do Revised Life Orientation Test (LOTR). Universitas Psychologica, Bogotá, v. 7, n. 2, p. 469-76, mai./ago. 2008.

LELOUP, J. Y. Além da luz e da sombra: sobre o viver, o morrer e o ser. 3 ed. Petrópolis: Vozes, 2001.

MCCOUCHLAN, M. A necessidade de cuidados paliativos. In: PESSINI, L.; BERTACHINI, L. (Org.). Humanização e cuidados paliativos. São Paulo: Loyola, 2004. p.161-18o.

PY, L.; OLIVEIRA, A. C. Humanizando o adeus à vida. In: PESSINI, L.; BERTACHINI, L. (Org.). Humanização e cuidados paliativos. São Paulo: Loyola, 2004. p. 135-48.

SANTOS, F. S. Tanatologia - a ciência da educação para a vida. In: SANTOS, F. S. Cuidados paliativos: discutindo a vida, a morte e o morrer. São Paulo: Atheneu, 2009. p. 1-29.
SILVA, J. V.; KIMURA, M. Adaptação Cultural e Validação do Instrumento de Medida de Capacidades de Autocuidado "Appraisal of SelfCare Agency Scale”. 2002. 96p. Pesquisa - Escola de Enfermagem da Universidade de São Paulo, São Paulo.

SILVA, J. V.; SIMÕES, I. A. R. Os significados de boa morte ou morte digna: as representações sociais do paciente, familiar cuidador e profissionais da área de saúde de Pouso Alegre, MG. 2008. Dissertação (Mestrado em Bioética) Universidade do Vale do Sapucaí, Pouso Alegre, MG.

SILVA, J. V.; SIMÕES, I. A. R.. Os significados de boa morte e morte digna. In: SILVA, J. V. Bioética - visão multidimensional. São Paulo: Iátria, 2010. p. 123-29.

SIMÃO, A. B. et al . A atuação do serviço social junto a pacientes terminais: breves considerações. Serviço Social \& Sociedade, São Paulo, n. 102, jun. 2010. Disponível em <http://www.scielo. br/scielo.php?script=sci_arttext\&pid=So101$66282010000200009 \& \operatorname{lng}=p t \& n r m=i s o>$. Acesso em: 08 nov. 2011.

VILETE, L.; FIGUEIRA, I.; COUTINHO, E. Adaptação transcultural para o português do Social Phobia Inventory (SPIN) para utilização entre estudantes adolescentes. Revista de Psiquiatria do Rio Grande do Sul, Porto Alegre, RS 2006. Disponível em: <http://www.scielo. br/scielo.php?script=sci_arttext\&pid=So101$81082006000100006 \& \operatorname{lng}=e n \& n r m=i s o>$. Acesso em: 10 out. 2011.

WITTKOWSKI, J. The construction of the multidimensional orientation toward dying and death inventory (MODDI-F). Death Studies, Wursburg, v. 24, p. 479-95, 2001.

Recebido em: 01/03/20II

Reapresentado em: 21/11/20II

Aprovado em: 06/12/2011 